PROCEEDINGS OF THE

AMERICAN MATHEMATICAL SOCIETY

Volume 126, Number 3, March 1998, Pages 701-710

S 0002-9939(98)04294-4

\title{
FINITE EUCLIDEAN GRAPHS OVER RINGS
}

\author{
A. MEDRANO, P. MYERS, H. M. STARK, AND A. TERRAS
}

(Communicated by Dennis A. Hejhal)

\begin{abstract}
We consider graphs attached to $(\mathbb{Z} / q \mathbb{Z})^{n}$, where $q=p^{r}$, for an odd prime $p$, using an analogue of the Euclidean distance. These graphs are shown to be mostly non-Ramanujan, in contrast to the case of Euclidean graphs over finite fields.
\end{abstract}

\section{INTRODUCTION}

Finite symmetric spaces over fields $\mathbb{F}_{q}, q=p^{r}, p$ a prime, have been studied by many authors (see Terras [11]). However, only the paper of Angel et al. [1] addresses the question of what happens to the non-Euclidean upper half plane graphs over rings $\mathbb{Z} / q \mathbb{Z}, q=p^{r}, p$ an odd prime. Here we ponder the finite Euclidean graphs over these finite rings. At the end of this introduction comparisons will be made with finite Euclidean graphs over fields $\mathbb{F}_{q}$, which we considered in Medrano et al. [5].

Part of our motivation is to find finite analogues of the real Euclidean space $\mathbb{R}^{n}$, and of the harmonic analysis of functions on $\mathbb{R}^{n}$, as discussed in Terras [12], Ch. 1 . We want to know if replacing $\mathbb{R}$ by the field $\mathbb{Z} / q \mathbb{Z}$ is better or worse than replacing $\mathbb{R}$ by the ring $\mathbb{F}_{q}$, for $q=p^{r}, p$ an odd prime.

At the moment, our measure of "better" is connected with another quest - the quest to find new examples of Ramanujan graphs, as defined in Lubotzky, Phillips and Sarnak [4]. A connected $k$-regular graph is Ramanujan if for all eigenvalues $\lambda$ of the adjacency matrix with $|\lambda| \neq k$, we have

$$
|\lambda| \leq 2 \sqrt{k-1}
$$

See Li [2], Lubotzky [3] and Sarnak [9] for more information on Ramanujan graphs. These graphs are of interest to builders of communication networks because they have good expansion constants, which implies good transmission of information. Ramanujan graphs are of interest to number theorists because their Ihara zeta functions satisfy the Riemann hypothesis (see Stark and Terras [10]). They are also of interest to a random walker because they lead to speedy confusion (see Myers [6]).

An outline of the paper follows:

Theorem 2.1 relates the degree of the $\operatorname{graph} \bmod p^{r}$ to the degree of the corresponding graph $\bmod p$. Corollary 2.4 is the analogue of Theorem 2.1 for some

Received by the editors September 11, 1996.

1991 Mathematics Subject Classification. Primary 11T23; Secondary 05C25.

Key words and phrases. Euclidean graph, Ramanujan graph, Kloosterman sums over rings.

(C)1997 American Mathematical Society 
of the eigenvalues of the adjacency matrices of these graphs. Theorem 2.5 shows that when $p \nmid a$, the graphs $X_{p^{r}}(n, a)$ defined after equation (2.2) below are only Ramanujan when $r=n=2$ and $p=3$. Theorem 2.9 and Corollary 2.10 give formulas for all the eigenvalues of $X_{p^{r}}(n, a)$ when $p \nmid a, r \geq 2$. The formulas involve eigenvalues for $X_{p^{r-1}}(n, a)$ and Kloosterman sums over the multiplicative group $\mathbb{Z}_{p^{r}}^{*}$. These ring Kloosterman sums were evaluated by Salié [8]. Kloosterman sums are of interest to number theorists for many reasons. For example they are Fourier coefficients of the modular forms called Poincaré series (see Sarnak [9], Ch. 1).

Michelle Dedeo has considered the finite Euclidean graphs mod $2^{r}$ and found them to behave similarly to the graphs considered here except that they are bipartite.

Comparisons of Euclidean graphs over finite rings and fields.

Differences

1) Over fields the graphs are asymptotically Ramanujan (and exactly so half the time) as $q$ approaches infinity. See Medrano et al. [5]. Over rings except for the smallest case, the graphs (with unit distance parameter) are not Ramanujan. See Theorem 2.5.

2) Over fields in even dimensions, 0 is not in the spectrum of the adjacency operator. Over rings, 0 occurs as an eigenvalue with high multiplicity in general. See Theorem 2.9 and Corollary 2.10.

\section{Similarities}

1) In both cases, for a finite ring or field $R$ with $q$ elements and graphs with vertices in $R^{n}$ there are only 1 or 2 isomorphism classes of graphs as we vary the distance $a$ over units in $R$. See Proposition 4 and Theorem 5 in Medrano et al. [5].

2) In both cases the eigenvalues of the adjacency operators of Euclidean graphs are built up from Kloosterman sums over the units in $R$. See formula (11) of Medrano et al. [5] and Theorem 2.9 below.

\section{Finite Euclidean graphs OVER RINGS}

Let $\mathbb{Z}_{q}$ be the ring $\mathbb{Z} / q \mathbb{Z}, q=p^{r}, p$ a prime, $p \geq 3$. Suppose $r \geq 2$ mostly, since the case $r=1$ can be found in Medrano et al. [5]. The finite Euclidean space $\mathbb{Z}_{q}^{n}$ consists of column vectors $x$, with $j$ th entry $x_{j} \in \mathbb{Z}_{q}$. Define the distance between $x, y \in \mathbb{Z}_{q}^{n}$ by

$$
d(x, y)={ }^{t}(x-y) \cdot(x-y)=\sum_{j=1}^{n}\left(x_{j}-y_{j}\right)^{2} .
$$

Here ${ }^{t} x=$ transpose of $x$. This distance has values in $\mathbb{Z}_{q}$. It is a point-pair invariant; i.e. $d(x+u, y+u)=d(x, y)$, for all $x, y, u \in \mathbb{Z}_{q}^{n}$. It is also invariant under the finite orthogonal group

$$
O\left(n, \mathbb{Z}_{q}\right)=\left\{\left.g \in \mathbb{Z}_{q}^{n \times n}\right|^{t} g \cdot g=I\right\} .
$$

Given $a \in \mathbb{Z}_{q}$, define the Euclidean graph $X_{q}(n, a)$ as follows. The vertices are the vectors in $\mathbb{Z}_{q}^{n}$, and two vertices $x, y \in \mathbb{Z}_{q}^{n}$ are adjacent if $d(x, y)=a$.

A Cayley graph $X(G, S)$ for an additive group $G$ and a symmetric edge set

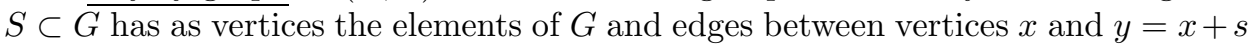
for $x, y \in G, s \in S$. The set $S$ is symmetric if $s \in S \Longrightarrow-s \in S$. If $S$ is a set of generators of $G$, the Cayley graph is connected. Usually this will be the case for our graphs. 
Let

$$
S_{q}(n, a)=\left\{x \in \mathbb{Z}_{q}^{n} \mid d(x, 0)=a\right\} .
$$

The Euclidean graph $X_{q}(n, a)$ is a Cayley graph for the additive group of $\mathbb{Z}_{q}^{n}$ with edge set $S_{q}(n, a)$.

Theorem 2.1. If $p \nmid a$, i.e. $a \in \mathbb{Z}_{q}^{*}=$ the multiplicative group of units $\bmod p$, the degree of the Euclidean graph $X_{p^{r}}(n, a)$ is given by

$$
\left|S_{p^{r}}(n, a)\right|=p^{(n-1)(r-1)}\left|S_{p}(n, a)\right| .
$$

Proof. Write ${ }^{t} x=\left(\begin{array}{lll}x_{1} & \cdots & x_{n}\end{array}\right)$, where $x_{j} \in \mathbb{Z}_{q}$, with $x_{j}=x_{j}^{(1)}+x_{j}^{(2)} p^{r-1}$ $\left(\bmod p^{r}\right)$, for $x_{j}^{(1)} \in \mathbb{Z}_{p^{r-1}}$ and $x_{j}^{(2)} \in \mathbb{Z}_{p}$. We also write $a=a^{(1)}+a^{(2)} p^{r-1}\left(\bmod p^{r}\right)$, for $a^{(1)} \in \mathbb{Z}_{p^{r-1}}$ and $a^{(2)} \in \mathbb{Z}_{p}$.

Then

$$
\sum_{j=1}^{n} x_{j}^{2}=\sum_{j=1}^{n}\left(x_{j}^{(1)}+x_{j}^{(2)} p^{r-1}\right)^{2} \equiv a^{(1)}+a^{(2)} p^{r-1}\left(\bmod p^{r}\right),
$$

iff

$$
\left\{\begin{array}{l}
\text { (i) } \sum_{j=1}^{n}\left(x_{j}^{(1)}\right)^{2}-a^{(1)}=p^{r-1} y, \\
\text { (ii) } y-a^{(2)}+2 \sum_{j=1}^{n} x_{j}^{(1)} x_{j}^{(2)} \equiv 0(\bmod p) .
\end{array}\right.
$$

If we find $x^{(1)} \in \mathbb{Z}_{p^{r-1}}^{n}$ solving (i), then we must solve (ii) for $x^{(2)} \in \mathbb{Z}_{p}^{n}$. Now (ii) is one linear equation in the unknown $x^{(2)}$. If $p \nmid a$, we know $x^{(1)}$ solving (i) will not vanish $\bmod p$. Then, the set of $x^{(2)}$ solving (ii) has order $p^{n-1}$ (take a particular solution of (ii) and add it to any solution of the homogeneous equation corresponding to (ii). It follows that

$$
\left|S_{a}^{(r)}\right|=p^{n-1}\left|S_{a}^{(r-1)}\right| .
$$

We get the desired result by induction on $r$.

Remark. In fact, for fixed $n$ and $q$ there are at most 2 non-isomorphic graphs $X_{q}(n, a)$ for $a$ not divisible by $p$. This is proved by the same method that proved Proposition 4 in Medrano et al. [5]. Moreover, when $n$ is even there is at most 1 non-isomorphic graph of this type by the proof of Theorem 5 in Medrano et al. [5].

Recall the formula for $\left|S_{p}(n, a)\right|$ from Medrano et al. [5]. When $p \nmid a$ we have

$$
\left|S_{p}(n, a)\right|=\left\{\begin{array}{c}
p^{n-1}+\chi\left((-1)^{\frac{n-1}{2}} a\right) p^{\frac{n-1}{2}}, n \text { odd }, \\
p^{n-1}-\chi\left((-1)^{\frac{n}{2}}\right) p^{\frac{n-2}{2}}, n \text { even. }
\end{array}\right.
$$

Here the Legendre symbol $\chi$ is defined by

$$
\chi(b)=\left\{\begin{array}{c}
1, \quad p \nmid b, b=\text { square } \bmod p, \\
-1, \quad p \nmid b, b \neq \text { square } \bmod p, \\
0, \quad p \mid b .
\end{array}\right.
$$


Our main goal is to study the spectrum of the adjacency operator $A_{a}$ acting on functions $f: \mathbb{Z}_{q}^{n} \longrightarrow \mathbb{C}$ by

$$
A_{a} f(x)=\sum_{d(x, y)=a} f(y)
$$

Define the exponentials

$$
\begin{aligned}
& e(v)=e^{(r)}(v)=\exp \left\{2 \pi i \frac{v}{p^{r}}\right\}, v \in \mathbb{Z}_{p^{r}}, \\
& e_{b}(u)=e_{b}^{(r)}(u)=\exp \left\{2 \pi i \frac{t}{p^{r} \cdot u}\right\}, b, u \in \mathbb{Z}_{q}^{n} .
\end{aligned}
$$

Proposition 2.2. The function $e_{b}$, for $b \in \mathbb{Z}_{q}^{n}$, defined by (2.8), is an eigenfunction of the adjacency operator $A_{a}$ of $X_{p^{r}}(n, a)$ corresponding to the eigenvalue

$$
\lambda_{b}^{(r)}=\sum_{d(s, 0)=a} e_{b}^{(r)}(s) .
$$

Moreover, as b runs through $\mathbb{Z}_{q}^{n}$, the $e_{b}(x)$ form a complete orthogonal set of eigenfunctions of $A_{a}$. Here we use the inner product on $f, g: \mathbb{Z}_{q}^{n} \longrightarrow \mathbb{C}$

$$
(f, g)=\sum_{x \in \mathbb{Z}_{q}^{n}} f(x) \overline{g(x)} .
$$

It follows that every eigenvalue of $X_{q}(n, a)$ has the form $\lambda_{b}$ for some $b \in \mathbb{Z}_{q}^{n}$.

Proof. The proof goes as in Medrano et al. [5], Proposition 2.2.

Note. As a corollary of Proposition 2.2 one can easily see that the multiplicity of the largest eigenvalue of the adjacency operator $(2.7)$ of $X_{q}(n, 1)$ is one. This implies that the graph is connected. One just needs to show that ${ }^{t} b s$ cannot be 0 for all $s \in S_{q}(n, 1)$, if $b \neq 0$. The key is that the unit vectors are in $S_{q}(n, 1)$. Of course, this fact shows directly that the set $S_{q}(n, 1)$ generates $\mathbb{Z}_{q}^{n}$ and, thus, that the graph is connected.

Theorem 2.3. Suppose $p \nmid a$, and $p \mid b_{j}$ for all $j=1, \ldots, n$. Then the eigenvalue $\lambda_{b}^{(r)}$ for $X_{q}(n, a)$ as in Proposition 2.2 is given by

$$
\lambda_{b}^{(r)}=p^{n-1} \lambda_{b / p}^{(r-1)}
$$

Proof. We use the same decomposition as in the proof of Theorem 2.1, as well as Proposition 2.2 above. Thus, by (2.4), we set

$$
A=\left\{s^{(1)} \in \mathbb{Z}_{p^{r-1}}^{n} \mid{ }^{t} s^{(1)} s^{(1)}-a^{(1)}=p^{r-1} y, \text { for some } y \in \mathbb{Z}\right\}
$$

and

$$
B=\left\{s^{(2)} \in \mathbb{Z}_{p}^{n} \mid 2^{t} s^{(1)} s^{(2)} \equiv a^{(2)}-y(\bmod p)\right\} .
$$

So we have

$$
\lambda_{b}^{(r)}=\sum_{s^{(1)} \in A} \sum_{s^{(2)} \in B} e^{(r)}\left({ }^{t} b^{(1)} s^{(1)}\right) e^{(1)}\left({ }^{t} b^{(1)} s^{(2)}\right) .
$$

Now, the inner sum consists of $s^{(2)}=s_{*}^{(2)}+h^{(2)}$, where $s_{*}^{(2)}$ is a particular solution of the inhomogeneous equation over $\mathbb{Z}_{p}$ defining $B$ in $(2.10)$, and $h^{(2)}$ solves the homogeneous equation

$$
{ }^{t} s^{(1)} h^{(2)} \equiv 0(\bmod p)
$$


Since $s^{(1)}$ is not equivalent to $0(\bmod p)$ (because $p \nmid a$, and $s^{(1)}$ satisfies the equation defining the set $A$ in $(2.9)$ ), we know that $h^{(2)}$ lies in an $(n-1)$-dimensional subspace of $\mathbb{Z}_{p}^{n}$.

Let $w_{1}, \ldots, w_{n-1}$ be a basis of the space of solutions $h^{(2)}$ of $(2.11)$. Then $h^{(2)}=$ $\sum_{j=1}^{n-1} u_{j} w_{j}, u_{j} \in \mathbb{Z}_{p}$ and since $p \mid b^{(1)}$,

$$
\sum_{h^{(2)}} e^{(1)}\left({ }^{t} b^{(1)} h^{(2)}\right)=p^{n-1} .
$$

Therefore

$$
\lambda_{b}^{(r)}=p^{n-1} \sum_{s^{(1)} \in A} e^{(r)}\left({ }^{t} b s^{(1)}\right)=p^{n-1} \lambda_{b / p}^{(r-1)} .
$$

Later (see Theorem 2.9 and Corollary 2.10) we will find another proof of Theorem 2.3 , but it is all we need to see that some of the eigenvalues are too large for our graphs to be Ramanujan, in most cases.

Corollary 2.4. Suppose that $p \nmid a$, and that $p^{r-1} \mid b_{j}$ for all $j=1, \ldots, n$. Then, using the notation of Theorem 2.3,

$$
\lambda_{b}^{(r)}=p^{(n-1)(r-1)} \lambda_{b / p^{r-1}}^{(1)} .
$$

Theorem 2.5. Suppose $p \nmid a$. Then, for $p \geq 3, r \geq 2, n \geq 2$, the graphs $X_{p^{r}}(n, a)$ are not Ramanujan, unless $p=3, r=2=n$.

Proof. From Angel et al. [1] let $k^{(r)}$ be the degree and $v^{(r)}$ be the number of vertices of $X_{p^{r}}(n, a)$. If $A^{(r)}$ is the adjacency operator on $X_{p^{r}}(n, a)$, let

$$
\mu^{(r)}=\max \left\{|\lambda| \mid \lambda \in \text { spectrum of } A^{(r)},|\lambda| \neq k^{(r)}\right\} .
$$

Then, by the inequality at the bottom of p. 455 of Angel et al. [1],

$$
\mu^{(r)} \geq \sqrt{\frac{k^{(r)} v^{(r)}-\left(k^{(r)}\right)^{2}}{v^{(r)}-1}} .
$$

Using Corollary 2.4 and (2.12) for $r=1$, we have that for the graphs $X_{p^{r}}(n, a)$

$$
\mu^{(r)} \geq p^{(n-1)(r-1)} \sqrt{\frac{k^{(1)} v^{(1)}-\left(k^{(1)}\right)^{2}}{v^{(1)}-1}},
$$

where $k^{(1)}$ is the degree $\left|S_{p}(n, a)\right|$ given in (2.5) and $v^{(1)}=p^{n}$. Set $k=k^{(1)}$ from now on. Also, Theorem 2.1 gives the Ramanujan bound for $X_{p^{r}}(n, a)$ which is $2 \sqrt{p^{(n-1)(r-1)} k-1}$.

So we want to show that (unless $p=3$ and $n=r=2$ ) we have:

$$
p^{(n-1)(r-1)} \sqrt{\frac{k p^{n}-k^{2}}{p^{n}-1}}>2 \sqrt{p^{(n-1)(r-1)} k-1} .
$$

This is equivalent to

$$
p^{(n-1)(r-1)} \frac{p^{n}-k}{p^{n}-1}>\frac{4\left(p^{(n-1)(r-1)} k-1\right)}{p^{(n-1)(r-1)} k} .
$$


Note that

$$
4 \geq \frac{4\left(p^{(n-1)(r-1)} k-1\right)}{p^{(n-1)(r-1)} k} .
$$

So it suffices to prove

$$
p^{(n-1)(r-1)}\left(p^{n}-k\right) \geq \dot{4} p^{n}-4 .
$$

From formula (2.5) we have four cases: $k=p^{n-1} \pm p^{\frac{n-1}{2}}$, and $k=p^{n-1} \pm p^{\frac{n-2}{2}}$. The worst case is $k=p^{n-1}+p^{\frac{n-1}{2}}$, so it suffices to substitute $k=p^{n-1}+p^{\frac{n-1}{2}}$ into (2.13) and show:

$$
p^{(n-1)(r-1)}\left(p^{n}-p^{n-1}-p^{\frac{n-1}{2}}\right)-\dot{4} p^{n}+4 \geq 0 .
$$

Case 1. First we will assume that $r \geq 2$ with $n \geq 2$ and $p \geq 7$ or $n \geq 3$ and $p \geq 3$. Set $r=2$ in (2.14) and then let $x=p^{n-1}$. The inequality becomes

$$
(p-1)\left(x^{2}-4 x\right)-x^{3 / 2}-4 x+4 \geq 0 .
$$

This holds for $n \geq 2$ and $p \geq 7$ or for $n \geq 3$ and $p \geq 3$.

Case 2. Our second case is $r \geq 3$, with $n \geq 2$ and $p=3$, 5. Set $r=3$ in (2.14) and then let $x=p^{n-1}$. Thus we must check

$$
(p-1)\left(x^{3}-4 x\right)-x^{5 / 2}-4 x+4 \geq 0 .
$$

This is easily checked for $n \geq 2, p=3,5$.

Case 3. The remaining cases $r=2=n$ and $p=3,5$ can be checked by computer. See the examples at the end of the paper.

In the rest of this paper, we want to derive a simple formula for the eigenvalues of $X_{q}(n, a)$ in the spirit of formula (11) of Medrano et al. [5]. Before beginning this discussion, we need to consider Gauss and Kloosterman sums over rings. These are finite analogues of gamma and Bessel functions. For $v \in \mathbb{Z}_{q}^{*}$ define the Gauss sum

$$
G_{v}^{(r)}=\sum_{y \in \mathbb{Z}_{q}} e\left(v y^{2}\right) .
$$

Then the method of proof of Theorems 2.1 and 2.3 leads to the following results.

Proposition 2.6. $G_{v}^{(r)}=p G_{v}^{(r-2)}$.

Corollary 2.7. $G_{v}^{(r)}=\left\{\begin{array}{c}p^{\frac{r}{2}}, r \text { even, } \\ p^{\frac{r-1}{2}} G_{v}^{(1)}, r \text { odd. }\end{array}\right.$

Now, $G_{v}^{(1)}=\chi(v) G_{1}^{(1)}$, where $\chi(v)$ is the Legendre symbol defined by $(2.6)$. Recall Gauss's formula

$$
G_{1}^{(1)}=\sqrt{p}\left\{\begin{array}{l}
1, p \equiv 1(\bmod 4) \\
i, p \equiv 3(\bmod 4)
\end{array} .\right.
$$

This is not the only kind of Gauss sum associated with rings. Another sort of Gauss sum over rings appears in Odoni [7].

Next we consider Kloosterman sums over rings. The main results are to be found in Salié [8]. See also Whiteman [13] and Williams [14] and [15]. If $\kappa$ is a character of the multiplicative group $\mathbb{Z}_{q}^{*}$ define the Kloosterman sum

$$
K^{(r)}(\kappa \mid a, b)=\sum_{t \in \mathbb{Z}_{q}^{*}} \kappa(t) e^{(r)}(a t+b \bar{t}) .
$$


Here $\bar{t}$ is the multiplicative inverse of $t \bmod q$; i.e., $t \bar{t} \equiv 1(\bmod q)$.

Proposition 2.8 (Salié [8]). Assume $r \geq 2$ and $p$ odd. Then using the notation of (2.17) we have the following evaluations of Kloosterman sums. Here $\chi$ denotes the Legendre symbol (2.6).

(1) $K^{(r)}(\kappa \mid a c, b \bar{c})=\kappa(c)^{-1} K(\kappa \mid a, b)$, if $p \nmid c$.

(2) $K^{(r)}(1 \mid 1, b)=0$, if $b$ is not a square $\bmod q$ or if $p \mid b$.

(3) For $p$ which do not divide $c$, we have

$$
K^{(r)}\left(1 \mid 1, c^{2}\right)=2 p^{\frac{r}{2}}\left\{\begin{array}{c}
\cos \left(\frac{4 \pi c}{p^{r}}\right), r \text { even }, \\
\chi(c) \cos \left(\frac{4 \pi c}{p^{r}}\right), r \text { odd and } p \equiv 1(\bmod 4), \\
-\chi(c) \sin \left(\frac{4 \pi c}{p^{r}}\right), r \text { odd and } p \equiv 3(\bmod 4) .
\end{array}\right.
$$

(4) $K(\chi \mid 1, b)=0$, if $b$ is not a square $\bmod q$ or if $p \mid b$ and $r$ is odd (we do not need to consider the case that $p \mid b$ and $r$ even).

(5) If $p \nmid c$, then

$$
K\left(\chi \mid 1, c^{2}\right)=2 p^{\frac{r}{2}} \cos \left(\frac{4 \pi c}{p^{r}}\right)\left\{\begin{array}{l}
1, p \equiv 1(\bmod 4), \\
i, p \equiv 3(\bmod 4) .
\end{array}\right.
$$

Theorem 2.9. Suppose $p \nmid a$ and $q=p^{r}$. Then we have the following formula for the eigenvalue $\lambda_{2 b}^{(r)}$ of the Euclidean graph $X_{q}(n, a): q \lambda_{2 b}^{(r)}=\sum_{1}+\sum_{2}$, where

$$
\sum_{1}=\left\{\begin{array}{l}
0, \text { if } p \nmid b_{j} \text { for some } j, \\
p^{r+n-1} \lambda_{2 b / p}^{(r-1)}, \text { if } p \mid b_{j} \text { for all },
\end{array}\right.
$$

and

$$
\sum_{2}=\sum_{v \in \mathbb{Z}_{q}^{*}}\left(G_{v}^{(r)}\right)^{n} e^{(r)}\left(-a v-\frac{1}{v}{ }^{t} b \cdot b\right) .
$$

Proof. We imitate the proof of formula (11) in Medrano et al. [5]:

$$
\begin{aligned}
q \lambda_{2 b} & =\sum_{x \in \mathbb{Z}_{q}^{n}} \sum_{v \in \mathbb{Z}_{q}} e^{(r)}\left(2^{t} b \cdot x+v\left({ }^{t} x \cdot x-a\right)\right) \\
& =\sum_{v \in \mathbb{Z}_{q}} e(-a v) \sum_{x \in \mathbb{Z}_{q}^{n}} e\left(2^{t} b \cdot x+v^{t} x \cdot x\right)=\sum_{1}+\sum_{2}
\end{aligned}
$$

where

$$
\sum_{1}=\sum_{p \mid v} e(-a v) \sum_{x \in \mathbb{Z}_{q}^{n}} e\left(2{ }^{t} b \cdot x+v^{t} x \cdot x\right)
$$

and

$$
\sum_{2}=\sum_{p \nmid v} e(-a v) \sum_{x \in \mathbb{Z}_{q}^{n}} e\left(2^{t} b \cdot x+v^{t} x \cdot x\right) .
$$

To evaluate $\sum_{1}$, set $v=p \cdot k, k \bmod p^{r-1}$ to obtain

$$
\sum_{1}=\sum_{x \in \mathbb{Z}_{q}^{n}} e\left(2^{t} b \cdot x\right)\left\{\sum_{k=0}^{p^{r-1}-1} e^{\frac{2 \pi i}{p^{r-1}}\left({ }^{t} x \cdot x-a\right) \cdot k}\right\} .
$$


Set $x_{j}=x_{j}^{(1)}+p^{r-1} x_{j}^{(2)}$, with $x_{j}^{(1)} \in \mathbb{Z}_{p^{r-1}}$, and $x_{j}^{(2)} \in \mathbb{Z}_{p}$. The inner sum is 0 unless ${ }^{t} x^{(1)} x^{(1)} \equiv a\left(\bmod p^{r-1}\right)$. And $x^{(2)}$ is arbitrary $\bmod p$, for note that

$$
{ }^{t} x x-a \equiv{ }^{t} x^{(1)} x^{(1)}-a\left(\bmod p^{r-1}\right) .
$$

So writing $b=b^{(1)}+p^{r-1} b^{(2)}$, with $b^{(1)} \in \mathbb{Z}_{p^{r-1}}, b^{(2)} \in \mathbb{Z}_{p}$, we have

$$
\sum_{1}=p^{r-1} \sum_{{ }^{t} x^{(1)} x^{(1)} \equiv a\left(\bmod p^{r-1}\right)} e^{\frac{4 \pi i}{p r}\left({ }^{t} b x^{(1)}\right)}\left\{\sum_{x^{(2)}} e^{\frac{4 \pi i}{p}\left(b^{(1)} x^{(2)}\right)}\right\}
$$

which has the stated value.

To evaluate $\sum_{2}$, we sum over $v$ such that $p \nmid v$. So we know that $v$ has an inverse $\bar{v} \bmod q$ as in (2.17) and we can complete the square to obtain

$$
\begin{aligned}
\sum_{2} & =\sum_{p \nmid v} e(-a v) \sum_{x \in \mathbb{Z}_{q}^{n}} e\left(v^{t}(x+b \bar{v}) \cdot(x+b \bar{v})\right) e\left(-\bar{v}^{t} b \cdot b\right) \\
& =\sum_{p \nmid v} e\left(-a v-{ }^{t} b \cdot b \bar{v}\right) \sum_{y \in \mathbb{Z}_{q}^{n}} e\left(v^{t} y \cdot y\right) \\
& =\sum_{p \nmid v}\left(G_{v}^{(r)}\right)^{n} e\left(-a v-{ }^{t} b \cdot b \bar{v}\right) .
\end{aligned}
$$

We can use the preceding results to get even more explicit formulas for the eigenvalues.

Corollary 2.10. Using the notation of Theorem 2.9, we have the following formulas for $\sum_{2}$. Here $\chi$ denotes the Legendre symbol (2.6).

(1) If $r$ is even,

$$
\sum_{2}=p^{\frac{r n}{2}}\left\{\begin{array}{l}
0, \text { if } a^{t} b b \neq s q u a r e \bmod q, \text { or if } p \mid a^{t} b b \\
2 p^{\frac{r}{2}} \cos \left(\frac{4 \pi c}{p^{r}}\right), \text { if } a^{t} b b=c^{2}, p \nmid c
\end{array}\right\} .
$$

(2) If $n$ is even and $r$ is odd,

$$
\sum_{2}=2 p^{\frac{r(n+1)}{2}} \chi(c)\left\{\begin{array}{l}
0, \text { if } a^{t} b b \neq \text { square }(\bmod q), \text { or } p \mid a^{t} b b, \\
\cos \left(\frac{4 \pi c}{p^{r}}\right), \text { if } a^{t} b b=c^{2}, p \nmid c, p \equiv 1(\bmod 4), \\
(-1)^{\frac{n}{2}+1} \sin \left(\frac{4 \pi c}{p^{r}}\right), \text { if } a^{t} b b=c^{2}, p \nmid c, p \equiv 3(\bmod 4) .
\end{array}\right.
$$

(3) If $n$ is odd and $r$ is odd,

$$
\sum_{2}=2 p^{\frac{r(n+1)}{2}} \chi(-\bar{a}) \cos \left(\frac{4 \pi c}{p^{r}}\right)\left\{\begin{array}{l}
0, \text { if } a^{t} b b \neq s q u a r e(\bmod q), \text { or } p \mid a^{t} b b, \\
1, \text { if } a^{t} b b=c^{2}, p \nmid c, p \equiv 1(\bmod 4), \\
(-1)^{\frac{n+1}{2}}, \text { if } a^{t} b b=c^{2}, p \nmid c, p \equiv 3(\bmod 4) .
\end{array}\right.
$$

Examples. The spectrum of eigenvalues (upper row) with multiplicities for $X_{9}(2,1)$ is

$$
\left(\begin{array}{lllllll}
-6 & -5.6382 & 0 & 1.0419 & 3 & 4.5963 & 12 \\
4 & 12 & 36 & 12 & 4 & 12 & 1
\end{array}\right)
$$


The Ramanujan bound $2 \sqrt{\text { degree }-1}$ for $X_{9}(2,1)$ is $2 \sqrt{11} \cong 6.633$ for $p=3$, which says $X_{9}(2,1)$ is a Ramanujan graph. And zero forms $\frac{36}{81} \cong 44 \%$ of the spectrum for $X_{9}(2,1)$.

The spectrum of eigenvalues (upper row) with multiplicities for $X_{25}(2,1)$ is

$$
\begin{aligned}
& \left(\begin{array}{lllllllll}
-16.1803 & -9.9211 & -9.2978 & -6.3742 & -5 & -4.2578 & -1.8738 & 0 \\
4 & 20 & 20 & 20 & 8 & 20 & 20 & 400
\end{array}\right) \\
& \left(\begin{array}{lllllllll}
.6279 & 1.9098 & 5.3583 & 6.1803 & 7.2897 & 8.7631 & 9.6858 & 13.0902 & 20 \\
20 & 4 & 20 & 4 & 20 & 20 & 20 & 4 & 1
\end{array}\right) .
\end{aligned}
$$

The Ramanujan bound for $X_{25}(2,1)$ is $2 \sqrt{19} \cong 8.718$, which says $X_{25}(2,1)$ is not Ramanujan. And zero forms $\frac{400}{625}=64 \%$ of the spectrum for $X_{25}(2,1)$.

It is easy to use Mathematica or Matlab to produce histograms of eigenvalues of these Euclidean graphs mod $q$ for small values of $q$. The shape of the histogram is very similar to that in Figure 2 of Angel et al. [1] giving the corresponding histogram for finite upper half planes over rings. These histograms also look somewhat similar to Figure 8 in Medrano et al. [5] giving the analogous histogram for a 3-dimensional Euclidean graph over the a finite field with 1019 elements. However, the spectrum of $X_{p^{2}}(2,1)$ appears to have gaps - unlike that for the Euclidean graphs over fields in 3 dimensions.

Those who like colorful computer graphics will note that there is no difference between Figures 4 and 5 in Terras [11] for prime or non-prime modulus $q$. The Mathematica command is:

$$
\begin{gathered}
\text { ListDensityPlot[Table }\left[\operatorname{Mod}\left[\mathrm{x}^{\wedge} 2+\mathrm{y}^{\wedge} 2, \mathrm{q}\right],\{\mathrm{x}, 1, \mathrm{q}\},\{\mathrm{y}, 1, \mathrm{q}\}\right], \\
\text { Mesh- }>\text { False,ColorFunction- }>\text { Hue }]
\end{gathered}
$$

\section{REFERENCES}

1. Jeff Angel, Bernadette Shook, Audrey Terras, and Cindy Trimble, Graph Spectra for Finite Upper Half Planes over rings, Linear Algebra Appl., 226-228 (1995), 423-457. MR 96g:05094

2. Wen-Ching Winnie Li, A survey of Ramanujan graphs, in R. Pellikaan, M. Perret and S.G. Vladut (Eds.), Arithmetic, Geometry and Coding Theory, Proc. Conf. at C.I.R.M., Luminy, June 28-July 2, 1993, de Gruyter, Berlin, 1996. CMP 96:14

3. Alexander Lubotzky, Discrete Groups, Expanding Graphs and Invariant Measures, Birkhäuser, Basel, 1994. MR 96g:22018

4. Alexander Lubotzky, Ralph Phillips, and Peter Sarnak, Ramanujan graphs, Combinatorica, 8 (1988), 261-277. MR 89m:05099

5. Archie Medrano, Perla Myers, Harold M. Stark, and Audrey Terras, Finite analogues of Euclidean space, J. Comput. Appl. Math. 68 (1996), 221-238. CMP 97:04

6. Perla Myers, Ph.D. Thesis, U.C.S.D., 1995.

7. R.W.K. Odoni, On Gauss sums $\left(\bmod p^{n}\right), n \geq 2$, Bull. London Math. Society, 5 (1973), 325327. MR 48:6020

8. Hans Salié, Über die Kloostermanschen Summen $S(u, v ; q)$, Math Z., 34 (1931), 91-109.

9. Peter Sarnak, Some Applications of Modular Forms, Cambridge U. Press, Cambridge, 1990. MR 92k: 11045

10. Harold M. Stark and Audrey Terras, Zeta functions of finite graphs and coverings, Advances in Math., 121 (1996), 124-165. CMP 96:15

11. Audrey Terras, Survey of spectra of Laplacians on finite symmetric spaces, Experimental Math., 5 (1996), 15-32. CMP 97:02

12. Audrey Terras, Harmonic Analysis on Symmetric Spaces and Applications, I,II, SpringerVerlag, New York, 1985, 1988. MR 87f:22010; MR 89k:22017 
13. Albert L. Whiteman, A note on Kloosterman sums, B.A.M.S., 51 (1945), 373-377. MR 6:259f

14. Kenneth S. Williams, Note on the Kloosterman sum, Proc. A.M.S., 30 (1971), 61-62. MR 44:2719

15. Kenneth S. Williams, The Kloosterman sum revisited, Canadian Math. Society Bull.,16 (1973), 363-365. MR 48:6021

(A. Medrano, H. M. Stark, and A. Terras) Department of Mathematics, University of California at San Diego, La Jolla, California 92093-0112

E-mail address: aterras@ucsd.edu

(P. Myers) Department of Mathematics, University of California, Santa Cruz, CaliFORNIA 95064

E-mail address: pmyers@cats.ucsc.edu 